\title{
Accuracy of quantitative vessel analysis in endovascular treatment for femoropopliteal lesions
}

\author{
Shinsuke Mori ${ }^{1}$, Keisuke Hirano ${ }^{2}$, Mitsuyoshi Takahara $^{3}$, Shigemitsu Shirai ${ }^{1}$, Kenji Makino ${ }^{1}$, \\ Yohsuke Honda ${ }^{1}$, Masakazu Tsutsumi ${ }^{1}$, Norihiro Kobayashi ${ }^{1}$, Masahiro Yamawaki ${ }^{1}$, Yoshiaki Ito ${ }^{1}$ \\ ${ }^{1}$ Department of Cardiology, Saiseikai Yokohama City Eastern Hospital, Yokohama, Japan; ${ }^{2}$ Department of Cardiology, Toyohashi Heart Center, \\ Toyohashi, Japan; ${ }^{3}$ Department of Diabetes Care Medicine, Osaka University Graduate School of Medicine, Suita, Japan \\ Contributions: (I) Conception and design: S Mori, K Hirano; (II) Administrative support: N Kobayashi, M Yamawaki, K Hirano, Y Ito; (III) Provision \\ of study materials or patients: S Mori, S Shirai, K Makino, Y Honda, M Tsutsumi, N Kobayashi; (IV) Collection and assembly of data: S Shirai, \\ K Makino, Y Honda; (V) Data analysis and interpretation: S Mori, M Tsutsumi; (VI) Manuscript writing: All authors; (VII) Final approval of \\ manuscript: All authors.
}

Correspondence to: Shinsuke Mori, MD. Department of Cardiology, Saiseikai Yokohama City Eastern Hospital, 3-6-1 Shimosueyoshi, Tsurumi-ku, Yokohama, Kanagawa 230-0012, Japan. Email: hello_morisun@yahoo.co.jp.

Background: Our aim was to evaluate the accuracy of quantitative vessel analysis (QVA) in measuring the reference vessel diameter (RVD) of femoropopliteal lesions.

Methods: Between October 2014 and September 2015, 30 consecutive femoropopliteal lesions in 25 patients who underwent endovascular therapy (EVT) under intravascular ultrasound (IVUS) guidance were analyzed. RVDs measured using $\mathrm{QVA}_{\text {sheath }}$ (calibrated using a 6-Fr sheath in the common femoral artery) and QVA $_{\text {ruler }}$ (calibrated using a ruler on the angiography table) were compared to those obtained using IVUS as the reference values.

Results: The mean $\mathrm{QVA}_{\text {sheath }}$-measured RVD was significantly larger than the mean IVUS-measured RVD

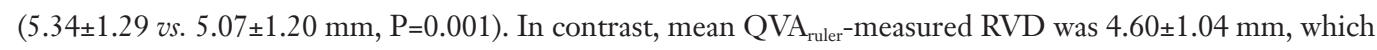
was significantly smaller than both the mean IVUS- and mean QVA sheath - measured RVD (both $\mathrm{P}<0.001$ ). Bland-Altman analysis revealed that the $95 \%$ limits of agreement versus IVUS ranged from -0.94 to $1.49 \mathrm{~mm}$ for $\mathrm{QVA}_{\text {sheath }}$ and -1.69 to $0.76 \mathrm{~mm}$ for $\mathrm{QVA}_{\text {ruler, }}$ respectively. Agreement with tolerance of $\pm 1.00 \mathrm{~mm}$ accounted for $88 \%$ of $\mathrm{QVA}_{\text {sheath }}$ and $83 \%$ of $\mathrm{QVA}_{\text {ruler }}(\mathrm{P}=0.60)$. The difference between QVA- and IVUSmeasured RVDs was inversely correlated with the distance from the table $\left(\mathrm{P}=0.029\right.$ for $\mathrm{QVA}_{\text {sheath }}$ and $\mathrm{P}=0.003$ for QVA ruler $_{\text {). }}$

Conclusions: The accuracy of both $\mathrm{QVA}_{\text {sheath }}$ and $\mathrm{QVA}_{\text {ruler }}$ in measuring RVD were similarly suboptimal. Over- and under-estimation of RVD is not rare in QVA.

Keywords: Quantitative vessel analysis (QVA); endovascular therapy (EVT); superficial femoral artery (SFA); intravascular ultrasound (IVUS); accuracy

Submitted Sep 26, 2020. Accepted for publication Jul 07, 2021.

doi: 10.21037/qims-20-1097

View this article at: https://dx.doi.org/10.21037/qims-20-1097 


\section{Introduction}

In the area of coronary angiography, quantitative coronary analysis (QCA) is used to assess the severity and progression of coronary artery disease, optimize device selection, and evaluate angiographic outcomes $(1,2)$. In contrast, quantitative vessel analysis (QVA) has not been established sufficiently for use in the femoropopliteal area. Several devices used for treating femoropopliteal lesions including stents and drug-coated balloons (DCB) come in sizes with gradations of $1 \mathrm{~mm}$; therefore, an accuracy of $\pm 1 \mathrm{~mm}$ is required for device selection when measuring vessel size in the femoropopliteal area. Previous reports have demonstrated various ways to calibrate an angiogram. Some of them include fixing a radiopaque ruler on a patient's upper thigh $(3,4)$, fixing a radiopaque ruler below a patient's upper thigh (5), and using a guide catheter or sheath (6-8). However, the accuracy of such methods remains unknown. Additionally, the vessel diameter appears to be influenced by the distance between the vessel and calibration point because they are not the same distance from the angiography table. Therefore, the aim of this study was to investigate the accuracy of QVAs in measuring the reference vessel diameter (RVD) and evaluate the relationship between the distance of a vessel from the angiography table and the accuracy of QVAs.

\section{Methods}

Between October 2014 and September 2015, 30 lesions in 25 consecutive patients who underwent endovascular therapy (EVT) for superficial femoral artery (SFA) lesions with intravascular ultrasound (IVUS) guidance were included in this study. Stent implantation was performed for de novo lesions and balloon dilatation was performed for in-stent restenosis lesions. Non-contrast lower extremity computed tomography $(\mathrm{CT})$ was also performed after the procedure to measure the distance between the angiography table and the treated blood vessel. Patients were excluded if they had acute or subacute lower limb ischemia and contraindications to angiography. All patients had symptoms after they received exercise and drug therapy. If angiography revealed stenosis $>50 \%$ of the diameter of the femoropopliteal artery, vascular specialists, including vascular surgeons and interventional cardiologists, decided on the applicability of EVT. This study was performed in accordance with the Declaration of Helsinki (as revised in 2013); it was approved by the ethics committee of our hospital and registered in the University Hospital Medical Information Network Clinical Trial
Registry (UMIN ID: 000016578). All patients provided written informed consent.

\section{Intervention}

EVTs were performed using the crossover approach. A 55-cm long, 6-Fr sheathless catheter (SheathLess PV; Asahi Intecc, Aichi, Japan) was inserted and unfractionated heparin (5,000 units) was injected intraarterially. Subsequently, a 0.014-inch guidewire was passed through the target lesion and balloon dilatation or stent implantation was performed. When two or more stents were used for a long lesion, the overlap was $\leq 10 \mathrm{~mm}$. Postdilatation was performed routinely in all lesions. At the end of the procedure, angiography was performed using a digital angiographic system (Allura Xper FD10 systems, Phillips Healthcare, Amsterdam, The Netherlands) and the original angiographic images were stored electronically. Angiography was performed with a radiopaque ruler under the thigh of the patient on the angiography table. Multiple planar images were not routinely obtained. IVUS was also performed, and the measuring points of IVUS were marked on angiographic images to compare the same point between IVUS and angiography. In all cases, IVUS images were recorded using a commercially available IVUS console (VISIWAVE ${ }^{\mathrm{TM}}$; Terumo Corporation, Tokyo, Japan) and a phased-array 35-MHz IVUS catheter (View IT; Terumo Corporation, Tokyo, Japan) as automatic pullback through the stented segment was performed at $2 \mathrm{~mm} / \mathrm{s}$. The working length of this IVUS is up to $15 \mathrm{~cm}$. When the stent length was more than $15 \mathrm{~cm}$, we performed automatic pullback of IVUS again from the beginning point of the second pullback according to the division of the ruler on the catheter table. All patients underwent non-contrast CT of the lower extremity within 2 days of the procedure.

\section{QVA}

Analysis of the angiographic data was performed using CAAS 5.7 (Pie Medical Imaging, Maastricht, The Netherlands). QVA was evaluated by two experienced observers and performed using two methods of calibration-a ruler along the femur on the catheter table and a 6-Fr sheathless catheter tip in the common femoral artery. The values of proximal and distal reference RVDs were measured. The proximal and distal RVDs selected for analysis were the most normal cross-sections within $10 \mathrm{~mm}$ of the proximal and distal margins of the stent. 


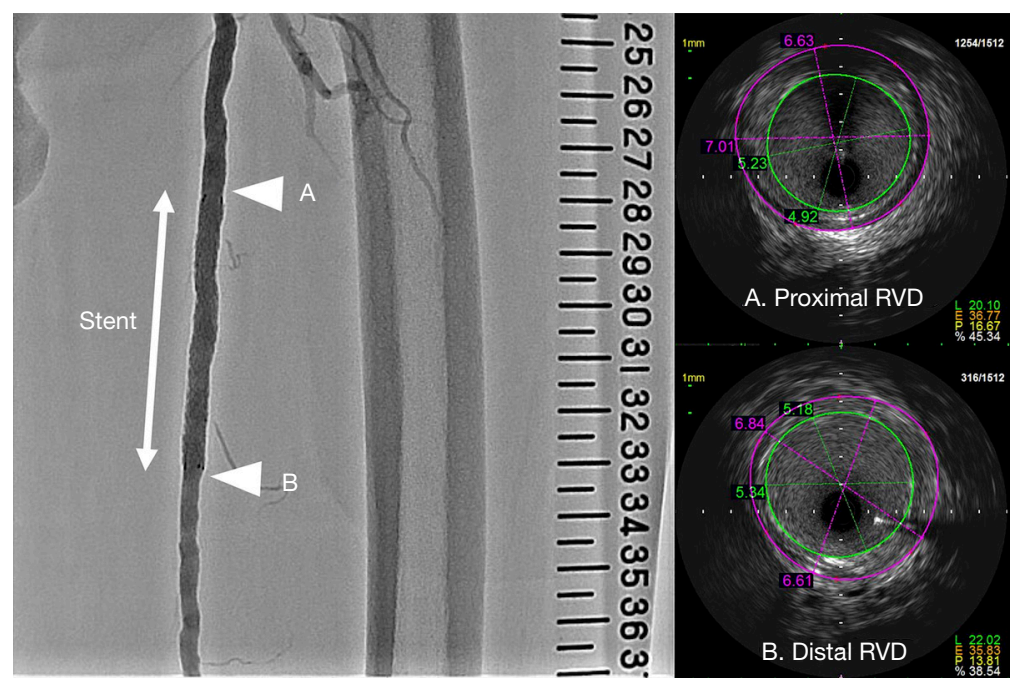

Figure 1 Angiography and IVUS analysis. A stent is placed at the part indicated by the arrow. A is the proximal end and B is the distal end. IVUS, intravascular ultrasound; RVD, reference vessel diameter.

\section{IVUS analysis}

For analysis of the IVUS data, VISIWAVE ${ }^{\mathrm{TM}}$ (Terumo Corporation) was used. Two experienced observers who were blinded to the angiographic findings performed IVUS analysis. The IVUS parameters measured or calculated were the proximal and distal reference lumen diameters (Figure 1).

\section{CT analysis}

Non-contrast CT of the lower extremity was performed using Aquilion 64-slice CT scanner (Canon Medical Systems, Japan). Data were acquired with collimation of $0.5 \times 64 \mathrm{~mm}$ and gantry rotation time of $500 \mathrm{~ms}$. The tube current was determined with auto exposure control at $100 \mathrm{kV}$, pitch value was 95.0 , and scan direction was craniocaudal. Ziostation 2 (Ziosoft Inc., Tokyo, Japan) was used to analyze the distance between the CT table and each of the stent edges. The distance from the CT table to each of the stent edges was substituted as the distance from the angiography table to each of the stent edges (Figure 2). All analyses were performed by two experienced observers who were blinded to the angiographic and IVUS findings.

\section{Vessel diameter analysis}

Vessel diameters obtained based on IVUS analysis were used as the standards for comparisons. The discrepancies in proximal and distal RVDs in $\mathrm{QVA}_{\text {sheath, }}, \mathrm{QVA}_{\text {ruler, }}$ and IVUS were evaluated.

\section{Inter-observer reproducibility}

In $\mathrm{QVA}_{\text {sheath }}$ measurements, the inter-observer agreement for RVD was 0.910 (95\% confidence interval: 0.849-0.946). In $\mathrm{QVA}_{\text {ruler }}$ measurements, the inter-observer agreement for RVD was $0.876(0.792-0.926)$. In IVUS measurements, the inter-observer agreement for RVD was 0.955 (0.915-0.976). In CT measurements, the inter-observer agreement for distance from the CT table to each of the stent edges was $0.998(0.997-0.999)$.

\section{Statistical analysis}

Data are expressed as mean \pm standard deviation for continuous variables and frequency (percentage) for discrete variables. $\mathrm{QVA}_{\text {sheath }}{ }^{-}$and $\mathrm{QVA}_{\text {ruler }}$-measured RVDs were compared with IVUS-measured RVD using Bland-Altman analysis (9). In brief, the differences between individual measurements of the two different measuring systems were calculated, and the means and standard deviations were derived. The $95 \%$ limits of agreement (i.e., $95 \%$ prediction intervals of the differences or errors) were obtained from the means and standard deviations. We also evaluated the proportions of the agreement with a tolerance of $\pm 1.00 \mathrm{~mm}$. We subsequently explored the association between the measurement difference and the distance from the 


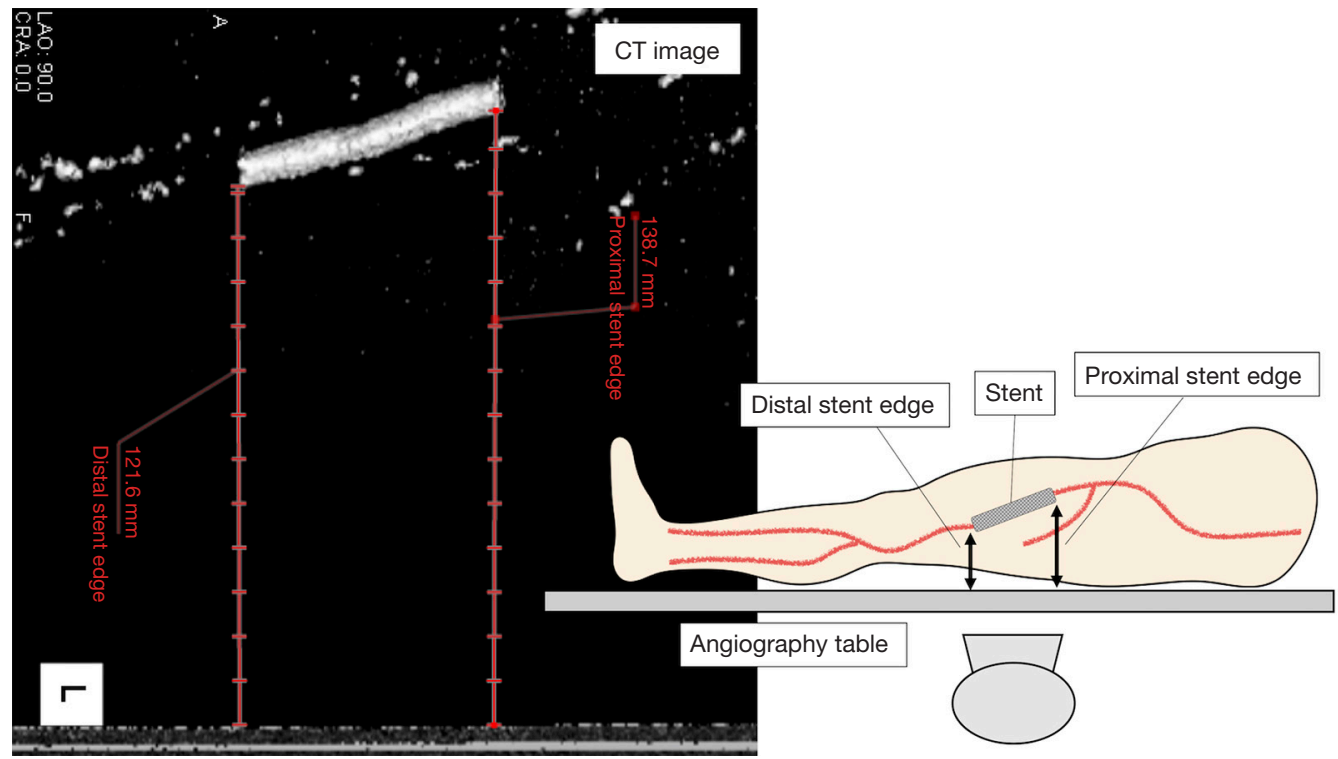

Figure 2 Analysis of height from angiography table to stent edge. The distance between the CT table and each the stent edges are analyzed with Ziostation 2. CT, computed tomography.

angiography table using Pearson's correlation analysis. All statistical analyses were performed and graphs were plotted using Microsoft Excel v2019 (Microsoft Corporation, Washington, USA).

\section{Results}

The characteristics of the study population are summarized in Table 1. The overall mean age was $76.2 \pm 8.4$ years and $36 \%$ of the study participants were female. The prevalence of diabetes mellitus, dialysis, and critical limb ischemia was $32 \%, 20 \%$, and $24 \%$, respectively. The rates of de novo lesions, Trans-Atlantic Inter-Society Consensus (TASC) II class $\mathrm{C}$ or D lesions, and popliteal lesions were $40 \%, 50 \%$, and $23 \%$, respectively. The mean IVUS-measured RVD was $5.07 \pm 1.20 \mathrm{~mm}$. The mean distance from the table was $117 \pm 26 \mathrm{~mm}$.

The mean QVA sheath -measured RVD was $5.34 \pm 1.29 \mathrm{~mm}$, which was significantly larger than the IVUS-measured RVD ( $\mathrm{P}=0.001$, paired $t$-test). In contrast, the mean $\mathrm{QVA}_{\text {ruler }}$-measured RVD was $4.60 \pm 1.04 \mathrm{~mm}$, which was significantly smaller than IVUS- and $\mathrm{QVA}_{\text {sheath }}{ }^{-}$ measured RVDs (both $\mathrm{P}<0.001$, paired $t$-test) (Figure 3). Figure 4 illustrates the Bland-Altman analysis between QVA- and IVUS-measured RVDs. The lower and upper $95 \%$ limits of agreement versus IVUS were -0.94 and $1.49 \mathrm{~mm}$ for $\mathrm{QVA}_{\text {sheath}}$, and -1.69 and $0.76 \mathrm{~mm}$ for $\mathrm{QVA}_{\text {ruler, }}$ respectively. The agreement with tolerance of $\pm 1.00 \mathrm{~mm}$ accounted for $88 \%$ (95\% confidence interval using ClopperPearson's exact method, 77-95\%) of $\mathrm{QVA}_{\text {sheath }}$ and $83 \%$ (71-92\%) for $\mathrm{QVA}_{\text {ruler }}(\mathrm{P}=0.60$, Fisher's exact test).

As illustrated in Figure 5, the difference between QVAand IVUS-measured RVD was inversely correlated with the distance from table.

\section{Discussion}

In QVA, the measured vessel diameter varies according to the calibration point and the source of $\mathrm{X}$-rays. It has been reported that a projected image of an object is affected by its distance between the calibration point and the source of X-rays. Takagi et al. reported that QCA is likely to overestimate the minimum stent diameter in the left circumflex artery (LCx) because LCx is anatomically closest to the $\mathrm{X}$-ray source (10). Similar principles apply to the femoropopliteal diameters; the arterial diameter varies according to the calibration point. Therefore, it can be inferred that vessel diameter may be both overestimated and underestimated by QVA.

Some previous studies have compared the assessment of vessel diameter of the femoropopliteal artery between QVA and IVUS. Pliagas et al. reported that angiographic imaging consistently underestimated the vessel size (11), and Arthurs et al. reported that angiography and IVUS provided similar 
Table 1 Baseline characteristics of the study population $(n=25)$

\begin{tabular}{|c|c|}
\hline Parameters & Value \\
\hline Age, years & $76.2 \pm 8.4$ \\
\hline Women, n [\%] & 9 [36] \\
\hline Body mass index, $\mathrm{kg} / \mathrm{m}^{2}$ & $22.7 \pm 4.5$ \\
\hline Hypertension, n [\%] & $21[84]$ \\
\hline Dyslipidemia, n [\%] & $10[40]$ \\
\hline Smoking, $\mathrm{n}$ [\%] & $6[24]$ \\
\hline Diabetes mellitus, $\mathrm{n}$ [\%] & 8 [32] \\
\hline Chronic kidney disease, n [\%] & $13[52]$ \\
\hline Dialysis, n [\%] & $5[20]$ \\
\hline Critical limb ischemia, $\mathrm{n}$ [\%] & $6[24]$ \\
\hline \multicolumn{2}{|l|}{ Lesion characteristics $(n=30)$} \\
\hline Ankle brachial index & $0.63 \pm 0.29$ \\
\hline De novo lesion, $\mathrm{n}[\%]$ & $12[40]$ \\
\hline TASC II class C/D, n [\%] & $15[50]$ \\
\hline Calcified lesion, n [\%] & $3[10]$ \\
\hline Involving popliteal lesion, n [\%] & 7 [23] \\
\hline Poor runoff, n [\%] & $13[43]$ \\
\hline IVUS-measured RVD, mm & $5.07 \pm 1.20$ \\
\hline Distance from table, $\mathrm{mm}$ & $117 \pm 26$ \\
\hline
\end{tabular}

TASC, Trans-Atlantic Inter-Society Consensus; IVUS, intravascular ultrasound; RVD, reference vessel diameter.

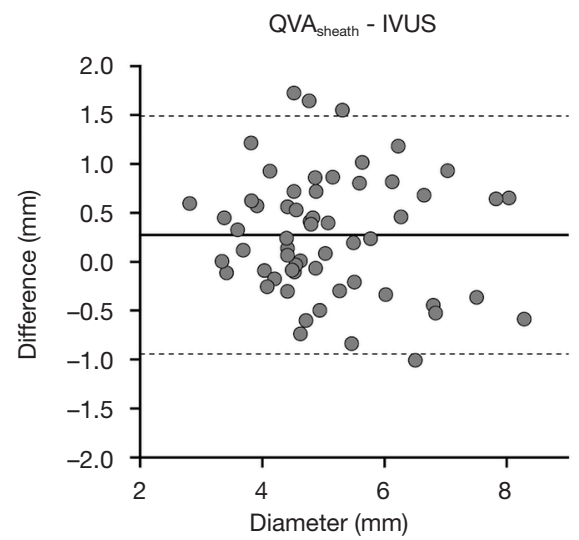

luminal diameters (12). In the present study, the mean $\mathrm{QVA}_{\text {sheath }}$-measured RVD was significantly larger than the IVUS-measured RVD $(5.34 \pm 1.29$ vs. $5.07 \pm 1.20 \mathrm{~mm}$, respectively, $\mathrm{P}=0.001$ ) and the mean $\mathrm{QVA}_{\text {ruler }}$-measured RVD was significantly smaller than the IVUS-measured RVD $(4.60 \pm 1.04$ vs. $5.07 \pm 1.20 \mathrm{~mm}$, respectively, $\mathrm{P}<0.001)$. These differences appear to have occurred because of differences in the QVA method used.

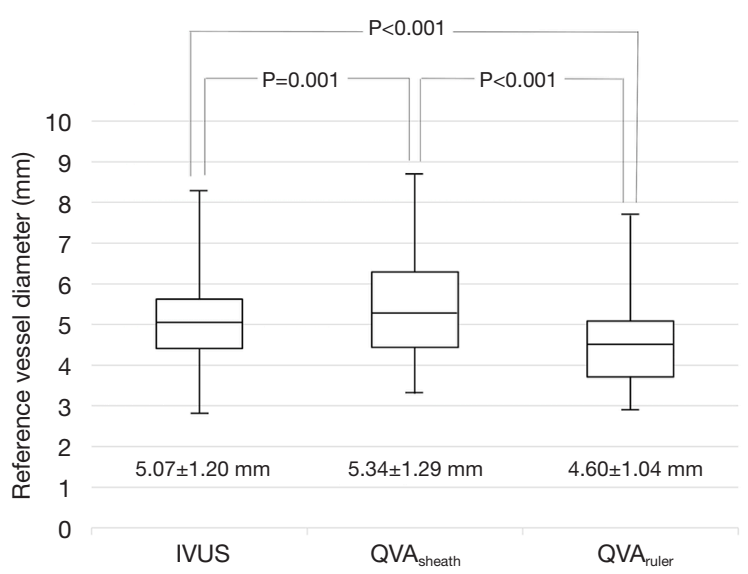

Figure 3 Comparison of RVD between IVUS, $\mathrm{QVA}_{\text {sheath, }}$, and QVA $_{\text {ruler }}$ The box plots represent RVD measured by IVUS, $\mathrm{QVA}_{\text {sheath }}$, and $\mathrm{QVA}_{\text {ruler }}$, respectively. RVD, reference vessel diameter; IVUS, intravascular ultrasound; QVA, quantitative vessel analysis.

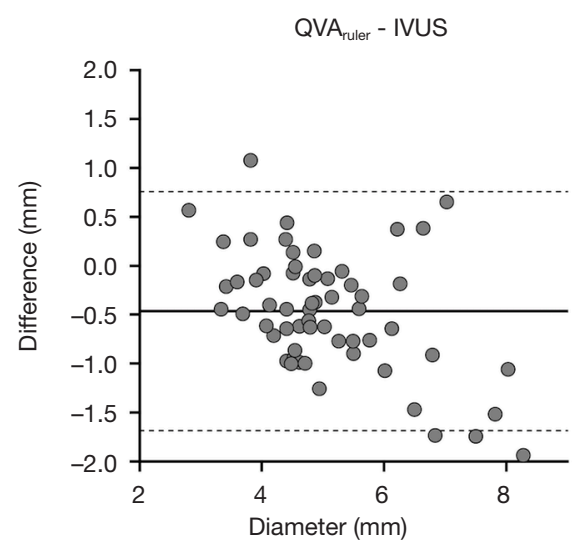

Figure 4 Differences between QVA- and IVUS-measured RVD. The horizontal axis represents IVUS-measured RVD and the vertical axis

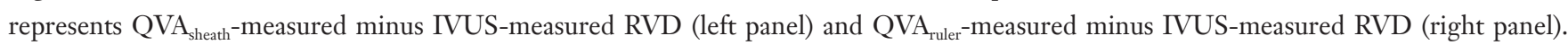
Thin dotted lines represent 95\% limits of agreement and bold sold lines demonstrate mean values. QVA, quantitative vessel analysis; IVUS, intravascular ultrasound; RVD, reference vessel diameter. 

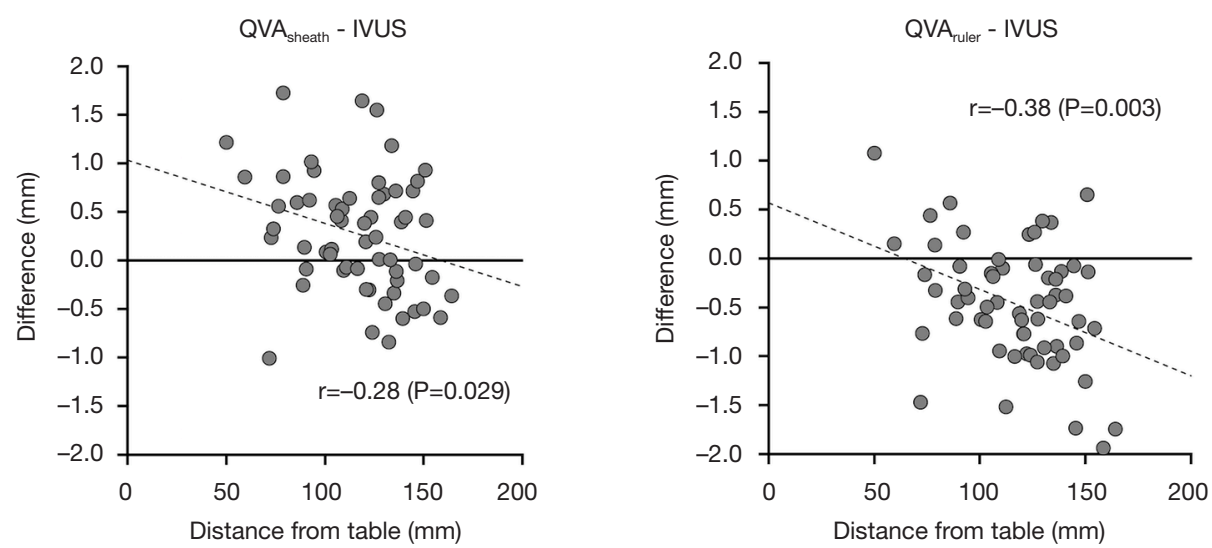

Figure 5 Correlation between height from angiography table and QVA-to-IVUS differences in RVD. Thin dotted lines represent the linear regression functions. QVA, quantitative vessel analysis; IVUS, intravascular ultrasound; RVD, reference vessel diameter.

Furthermore, the findings of the present study, in which femoropopliteal segments were evaluated, provide additional and new information regarding the level of expected error with $\mathrm{QVA}_{\text {sheath }}$ and $\mathrm{QVA}_{\text {ruler }}$ compared with IVUS. BlandAltman analysis between QVA- and IVUS-measured RVDs revealed that the lower and upper $95 \%$ limits of agreement versus IVUS were -0.94 and $1.49 \mathrm{~mm}$ for $\mathrm{QVA}_{\text {sheath }}$ and -1.69 and $0.76 \mathrm{~mm}$ for $\mathrm{QVA}_{\text {ruler }}$ respectively. The agreement with a tolerance of $\pm 1.00 \mathrm{~mm}$ accounted for $88 \%$ (77-95\%) of $\mathrm{QVA}_{\text {sheath }}$ and $83 \%$ (71-92\%) of $\mathrm{QVA}_{\text {ruler }}$; however, there was no significant difference in the accuracy between $\mathrm{QVA}_{\text {ruler }}$ and $\mathrm{QVA}_{\text {sheath }}(\mathrm{P}=0.60)$. Therefore, more than $1 \mathrm{~mm}$ of discrepancy can occur in as high as $12 \%(5-23 \%)$ of cases with QVA $_{\text {sheath }}$ and 17\% (8-29\%) of cases with QVA $_{\text {ruler; }}$; over- and under-estimation of RVD is not rare with QVA, irrespective of the method used. This inherent issue might result in selecting oversized or undersized devices compared with the actual vessel size.

Undersized balloon dilatation may result in suboptimal vessel expansion and insufficient gains in the lumen. Undersized DCB may result in the lack of apposition between the balloon and the vessel wall and insufficient drug delivery to the tissue, which may contribute to poor clinical outcomes. Undersized stents may result in stent malapposition and risks of restenosis and late thrombosis. In contrast, oversized balloon dilatation may result in severe vessel dissection, and the opportunity of drug balloon angioplasty may be lost. It has been reported that oversized stents were related to in-stent restenosis following self-expandable stenting for femoropopliteal lesions (6). Oversized interwoven nitinol biomimetic Supera stents
(Abbott Vascular, Santa Clara, CA, USA) cause elongation and increases the rate of restenosis (13). Therefore, it is crucial to choose correctly sized devices to improve the clinical outcomes of EVT. The findings of this study demonstrate that QVA does not always guarantee accurate estimation of RVD in the selection of device size. IVUS enables choosing accurately sized devices. On the other hand, whether routine IVUS usage during EVT would lead to improvements in treatment of femoropopliteal lesions remains unclear. Further research is needed in this area.

The discrepancy between measuring methods correlates to the distance from the angiography table. For $\mathrm{QVA}_{\text {ruler, }}$, the further the distance from the table, the greater the underestimation of the vessel diameter. In contrast, for $\mathrm{QVA}_{\text {sheath }}$, the vessel diameter was overestimated. A significantly large error can appear in people with a larger body size as well. As such, these factors should be recognized when estimating vessel diameter for EVT of femoropopliteal lesions.

There were some limitations to this study. First, this study included a small number of patients. Second, angiography was performed in only one direction, which can possibly increase the estimation of the error; however, angiography in two directions is not usually performed in routine practice. Third, diameter measurement value on angiographic images can be influenced by many factors such as the injection speed and volume thus to some extent. Forth, the distance from the CT table to each of the stent edges was substituted as the distance from the angiography table to each of the stent edges in this study. There may be a slight error between them because the site measured during EVT cannot be 
accurately located on non-contrast CT images. Finally, QVA with calibration using a ruler on the thigh was not evaluated in this study because the ruler in such cases is not along the horizontal plane. Further studies are required to explore this approach and corroborate our findings.

\section{Conclusions}

The accuracies of $\mathrm{QVA}_{\text {sheath }}$ and $\mathrm{QVA}_{\text {ruler }}$ in measuring RVD were similarly suboptimal. Over- and under-estimation of RVD is not rare in QVA.

\section{Acknowledgments}

Funding: None.

\section{Footnote}

Conflicts of Interest: All authors have completed the ICMJE uniform disclosure form (available at https://dx.doi. org/10.21037/qims-20-1097). The authors have no conflicts of interest to declare.

Ethical Statement: The autors are accountable for all aspects of the work in ensuring that questions related to the accuracy or integrity of any part of the work are appropriately investigated and resolved. This study was performed in accordance with the Declaration of Helsinki (as revised in 2013); it was approved by the ethics committee of our hospital and registered in the University Hospital Medical Information Network Clinical Trial Registry (UMIN ID: 000016578). All patients provided written informed consent.

Open Access Statement: This is an Open Access article distributed in accordance with the Creative Commons Attribution-NonCommercial-NoDerivs 4.0 International License (CC BY-NC-ND 4.0), which permits the noncommercial replication and distribution of the article with the strict proviso that no changes or edits are made and the original work is properly cited (including links to both the formal publication through the relevant DOI and the license). See: https://creativecommons.org/licenses/by-nc-nd/4.0/.

\section{References}

1. Moses JW, Leon MB, Popma JJ, Fitzgerald PJ, Holmes DR, O'Shaughnessy C, Caputo RP, Kereiakes DJ,
Williams DO, Teirstein PS, Jaeger JL, Kuntz RE; SIRIUS Investigators. Sirolimus-eluting stents versus standard stents in patients with stenosis in a native coronary artery. N Engl J Med 2003;349:1315-23.

2. Regar E, Serruys PW, Bode C, Holubarsch C, Guermonprez JL, Wijns W, Bartorelli A, Constantini C, Degertekin M, Tanabe K, Disco C, Wuelfert E, Morice MC; RAVEL Study Group. Angiographic findings of the multicenter Randomized Study With the SirolimusEluting Bx Velocity Balloon-Expandable Stent (RAVEL): sirolimus-eluting stents inhibit restenosis irrespective of the vessel size. Circulation 2002;106:1949-56.

3. Amighi J, Schillinger M, Dick P, Schlager O, Sabeti S, Mlekusch W, Haumer M, Mathies R, Heinzle G, Schuster A, Loewe C, Koppensteiner R, Lammer J, Minar E, Cejna $M$. De novo superficial femoropopliteal artery lesions: peripheral cutting balloon angioplasty and restenosis rates-randomized controlled trial. Radiology 2008;247:267-72.

4. Schillinger M, Sabeti S, Loewe C, Dick P, Amighi J, Mlekusch W, Schlager O, Cejna M, Lammer J, Minar E. Balloon angioplasty versus implantation of nitinol stents in the superficial femoral artery. $\mathrm{N}$ Engl J Med 2006;354:1879-88.

5. Krankenberg H, Schlüter M, Steinkamp HJ, Bürgelin K, Scheinert D, Schulte KL, Minar E, Peeters P, Bosiers M, Tepe G, Reimers B, Mahler F, Tübler T, Zeller T. Nitinol stent implantation versus percutaneous transluminal angioplasty in superficial femoral artery lesions up to 10 $\mathrm{cm}$ in length: the femoral artery stenting trial (FAST). Circulation 2007;116:285-92.

6. Soga Y, Yokoi H, Urakawa T, Iwabuchi M, Nobuyoshi M. Clinical impact of self-expandable stent diameter after femoropopliteal stenting. Cardiovasc Interv Ther 2011;26:38-44.

7. Pavlovic C, Futamatsu H, Angiolillo DJ, Guzman LA, Wilke N, Siragusa D, Wludyka P, Percy R, Northrup M, Bass TA, Costa MA. Quantitative contrast enhanced magnetic resonance imaging for the evaluation of peripheral arterial disease: a comparative study versus standard digital angiography. Int J Cardiovasc Imaging 2007;23:225-32.

8. Duda SH, Pusich B, Richter G, Landwehr P, Oliva VL, Tielbeek A, Wiesinger B, Hak JB, Tielemans H, Ziemer G, Cristea E, Lansky A, Bérégi JP. Sirolimus-eluting stents for the treatment of obstructive superficial femoral artery disease: six-month results. Circulation 2002;106:1505-9.

9. Bland JM, Altman DG. Statistical methods for assessing agreement between two methods of clinical measurement. 
Lancet 1986;1:307-10.

10. Takagi K, Shannon J, Basavarajaiah S, Latib A, Al-Lamee R, Hasegawa T, Godino C, Ferraro M, Figini F, Carlino M, Montorfano M, Chieffo A, Colombo A. Discrepancies in vessel sizing between angiography and intravascular ultrasound varies according to the vessel evaluated. Int J Cardiol 2013;168:3791-6.

11. Pliagas G, Saab F, Stavroulakis K, Bisdas T, Finton S, Heaney C, McGoff T, Hardy K, Adams G, Mustapha JA. Intravascular ultrasound imaging versus digital subtraction angiography in patients with peripheral vascular disease. J
Invasive Cardiol 2020;32:99-103.

12. Arthurs ZM, Bishop PD, Feiten LE, Eagleton MJ, Clair DG, Kashyap VS. Evaluation of peripheral atherosclerosis: a comparative analysis of angiography and intravascular ultrasound imaging. J Vasc Surg 2010;51:933-8; discussion 939.

13. Garcia LA, Rosenfield KR, Metzger CD, Zidar F, Pershad A, Popma JJ, Zaugg M, Jaff MR; SUPERB investigators. SUPERB final 3-year outcomes using interwoven nitinol biomimetic supera stent. Catheter Cardiovasc Interv 2017;89:1259-67.

Cite this article as: Mori S, Hirano K, Takahara M, Shirai S, Makino K, Honda Y, Tsutsumi M, Kobayashi N, Yamawaki M, Ito Y. Accuracy of quantitative vessel analysis in endovascular treatment for femoropopliteal lesions. Quant Imaging Med Surg 2022;12(1):568-575. doi: 10.21037/qims-20-1097 